\title{
Review
}

Fetal Diagnosis ant Therapy

\section{Mirror Syndrome: A Systematic Review of Fetal Associated Conditions, Maternal Presentation and Perinatal Outcome}

\author{
Thorsten Braun ${ }^{\mathrm{a}}$ Martin Brauer ${ }^{\mathrm{a}} \quad$ Ilka Fuchs $^{\mathrm{a}} \quad$ Christoph Czernik $^{\mathrm{b}}$ \\ Joachim Wolfram Dudenhausen ${ }^{\text {a }}$ Wolfgang Henrich $^{\text {a Nanette Sariogluc }}{ }^{c}$ \\ Departments of ${ }^{\mathrm{a}}$ Obstetrics, ${ }^{\mathrm{b}}$ Neonatology, and ${ }^{\mathrm{C}}$ Pathology, Charité Campus Virchow, Berlin, Germany
}

\section{Key Words}

Ebstein's anomaly $\cdot$ Mirror syndrome $\cdot$ Ballantyne

syndrome $\cdot$ Hydrops fetalis $\cdot$ Anemia $\cdot$ Maternal edema

\begin{abstract}
Introduction: Mirror syndrome, also referred to as Ballantyne's syndrome, is normally defined as the development of maternal edema in association with fetal hydrops. The incidence of mirror syndrome is low and few cases have been published. We describe a case report in association with fetal Ebstein anomaly and provide a systematic review on the fetal associated conditions, maternal presentation and perinatal outcome reported for mirror syndrome. Data Sources: A PubMed database search was done until December 2008 (English, French or German) without any restriction of publication date or journal, using the following key words: Ballantyne syndrome, Mirror syndrome, Triple edema, Pseudotoxemia, Maternal hydrops syndrome, Pregnancy toxemia, Acute second trimester gestosis, and Early onset preeclampsia. Reported cases were considered eligible when fetal associated conditions, maternal symptoms and fetal outcome were clearly described. Results: Among 151 publications a total of 56 reported cases satisfying all inclusion criteria were identified. Mirror syndrome was associated with rhesus isoimmunization (29\%), twin-twin transfusion syndrome (18\%),
\end{abstract}

viral infection (16\%) and fetal malformations, fetal or placental tumors (37.5\%). Gestational age at diagnosis ranged from 22.5 to 27.8 weeks of gestation. Maternal key signs were edema (80-100\%), hypertension (57-78\%) and proteinuria (20$56 \%$ ). The overall rate of intrauterine death was $56 \%$. Severe maternal complications including pulmonary edema occurred in $21.4 \%$. Maternal symptoms disappeared 4.8-13.5 days after delivery. Discussion: Mirror syndrome is associated with a substantial increase in fetal mortality and maternal morbidity.

Copyright $\odot 2010$ S. Karger AG, Basel

\section{Introduction}

John W. Ballantyne [1-3] in 1892 was the first to describe the association of maternal edema in pregnancy with fetal and placental hydrops due to rhesus isoimmunization [4]. Over the years this maternal pathology which 'mirrors' fetal pathology has been given various names, such as pseudotoxemia, maternal hydrops syndrome, pregnancy toxemia, acute second trimester gestosis, early onset preeclampsia, maternal hydrops syndrome, triple edema or mirror syndrome $[5,6]$. As it is uncommon and frequently underdiagnosed, the incidence of mirror syndrome is not clear. Prior to 2007 (less 

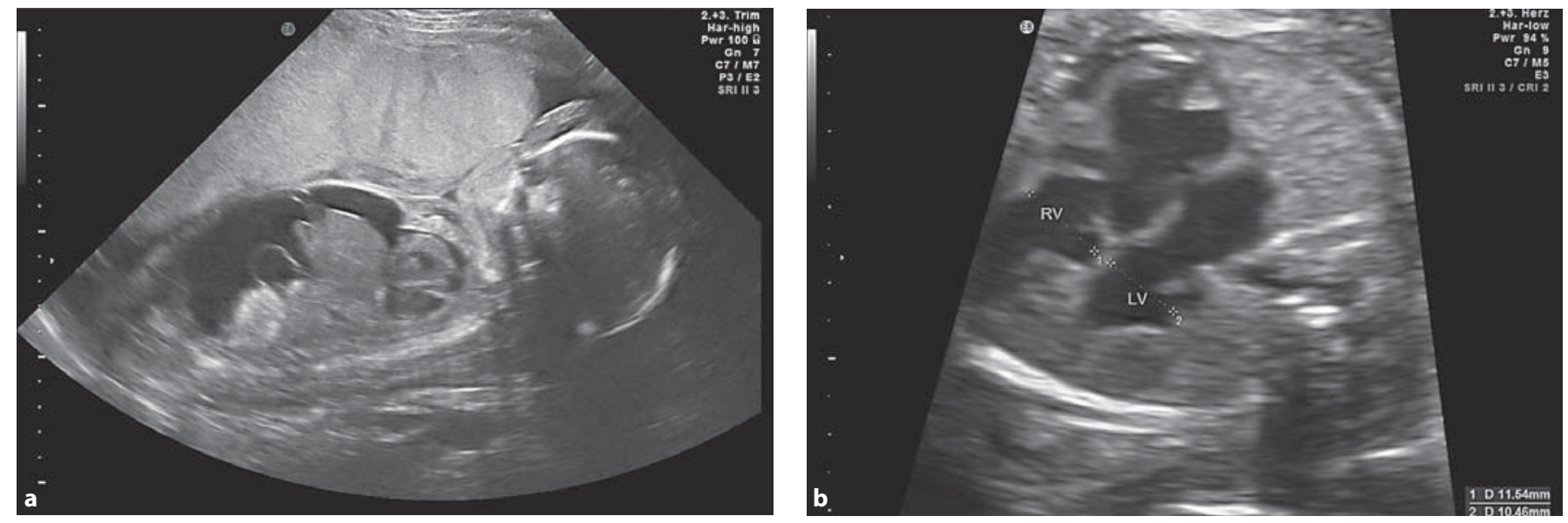

Fig. 1. a Ultrasonographic findings at 26 weeks 0 days of gestation of the placental edema; fetal hydrops with ascites and subcutaneous edema, pronounced at the forehead. b Ultrasonographic findings of the fetal echocardiography: cardiomegaly with biventricular myocardial hypertrophy; displacement of the tricuspid valve towards the apex of the right ventricle. $\mathrm{RV}=$ Right ventricle, $\mathrm{LV}=$ left ventricle.

than) 25 cases were reported $[7,8]$. The pathogenesis and pathophysiology is unknown [8]. In the late 1970s the development of ultrasound and prenatal diagnosis allowed description of cases of mirror syndrome in association with non-immunological, structural causes, for example aneurysm of Galen's vein [9], sacrococcygeal teratoma [10] or placental chorioangioma [11]. Non-structural causes such as fetal arrhythmias [12], human parvovirus infection $[7,13,14]$ and Coxsackie virus [15] infection were reported as well (table 1). Ebstein's anomaly occurs in $0.5 \%$ of patients with congenital heart disease with a sonographic typical distal displacement of the septal and posterior leaflets of the tricuspid valve. In severe cases this malformation is associated with hydrops fetalis and a very poor fetal prognosis $[6,16,17]$.

\section{Case Report}

A 27-year-old woman, gravida 1, para 1, group A, rhesus-negative was referred at 25 weeks 6 days of gestation to our department because of severe hydrops fetalis. Maternal examination revealed mild edema of the ankles and legs, normal blood pressure, minimal proteinuria (table 2). No blood group antibody was detected, TORCH serology was normal, amniocentesis showed a normal karyotype.

Fetal ultrasonic examination confirmed a massive ascites, subcutaneous edema, placental edema, pleural effusion and polyhydramnios (fig. 1a). Fetal echocardiography presented a cardiomegaly with biventricular myocardial hypertrophy and a severe Ebstein's anomaly: third-degree tricuspid insufficiency, displacement of the tricuspid valve towards the apex of the right ventricle, valve leaflets in part attached to the septal wall (fig. 1b). The right atrium was dilated to $17.5 \mathrm{~mm}$ with no evidence of blood flow via the foramen ovale. Truncus pulmonalis appeared to be normal, but without visualization of antegrade blood flow via the pulmonary valve. The perfusion of the pulmonary arteries seemed to be retrograde via the ductus arteriosus.

An intravenous digoxin therapy with an initial dose of $800 \mu \mathrm{g} /$ day, followed by an oral preservation therapy with methyldigoxin $\left(\right.$ Novodigal $^{\circledR}$ ) with a serum level of up to $2.5 \mu \mathrm{g} / \mathrm{l}$, was started to improve fetal cardiac function and to reduce the hydrops fetalis as reported before to be useful in Ebstein's anomaly [18]. Maternal echocardiography was normal at the time of admission.

After 3 days, the patient presented increased dyspnea, chest pain, mild fever and a need for oxygen. With pneumonia as the tentative diagnosis, combined antibiotic therapy was started. The patient developed massive generalized edema and total body weight increased $9 \mathrm{~kg}$ in 8 days. A repeated maternal echocardiotocography on day 7 presented an increased insufficiency of the mitral valve, tricuspid valve and pulmonary valve with biventricular increase of volume and pressure load and pericardial effusion. A spiral CT scan of the lungs completed the clinical diagnosis of a massive lung edema and pleural effusion. No signs of embolization of the pulmonary arteries were noted.

Fetal echocardiography itself presented a progress of hydrops fetalis. Fewer fetal movements were observed, pulsed-wave Doppler of the umbilical artery revealed increased pulsatility indices, and pulsed-wave Doppler of the middle cerebral artery presented a trend towards a brain-sparing effect. Fetal prognosis was estimated to be very poor by the obstetrical and pediatric staff due to congestive heart failure; discussion with the patient and her husband led to abstention. After induction with 4 doses of misoprostol $(4 \times 50$ $\mu$ C Cytotec $\left.^{\circledR}\right)$ the patient went into labor. At 26 weeks 6 days of gestation, a girl, 1,860 g, was spontaneously delivered alive but died 9 min after. The placenta was grossly edematous, weighed $885 \mathrm{~g}$ with no signs of chorioamnionitis. Fetal hemoglobin was $14.7 \mathrm{~g} / \mathrm{dl}$. 


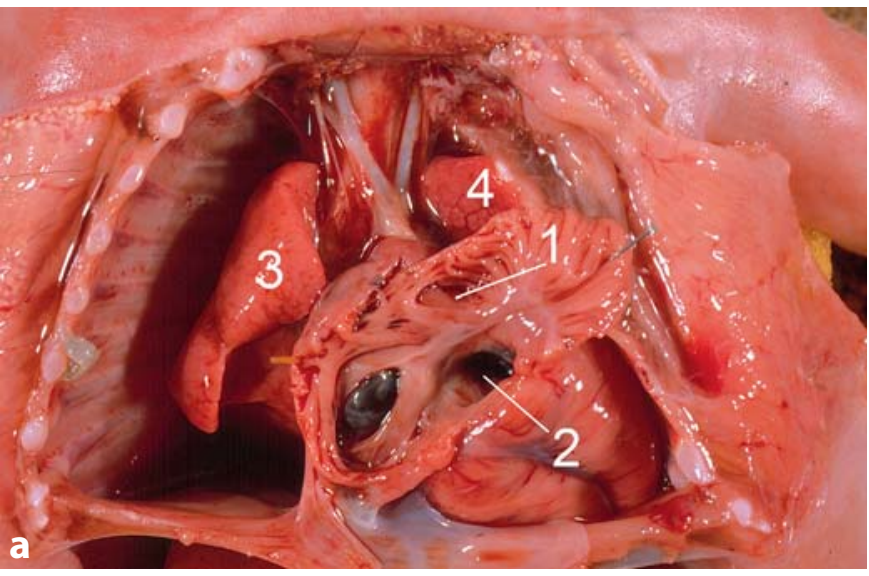

Fig. 2. a Autopsy images at 28 weeks of gestation. The heart is in situ. The right atrium is opened. View through the right atrioventricular junction into the right ventricle $(1=$ right atrium, $2=$ tricuspid valve, 3 = right lung, $4=$ left lung). b This heart has been opened through the right atrioventricular junction to show the typical feature of Ebstein's malformation. Displacement of the hinge point of the septal and dorsal leaflets of the tricuspid valve from the atrioventricular junction into the cavity of the right ventricle, the leaflets themselves being dysplastic. The foramen ovale is open but narrow ( 1 = right atrium, 2 = septal and dorsal leaflets of the tricuspid valve, 3 = foramen ovale).

The patient was discharged 7 days after delivery with normal cardiac function and well-being (weight loss $=17 \mathrm{~kg}$ ). Autopsy confirmed the prenatal findings and diagnosis of an Ebstein's anomaly; pulmonary cardiac failure due to lung hypoplasia and decompensated congenital cardiac anomaly caused fetal death (fig. 2).

Systematic Review of Mirror Syndrome

A PubMed database search in English, French or German was performed without any restriction of publication date or journal, using the following key words: Ballantyne syndrome, Mirror syndrome, Triple edema, Pseudotoxemia, Maternal hydrops syndrome, Pregnancy toxemia, Acute second trimester gestosis, and Early onset preeclampsia. The last search was updated in December 2008. All references of the selected articles were hand-searched for relevant studies not captured by electronic searches. Cases reported more than once by the same team in different publications were counted only once. Individually reported cases were not recounted in the larger case series. For each included case we focused on the following criteria: time of diagnosis, time of antenatal symptoms, time of delivery, time until maternal reconvalescence, maternal symptoms (weight gain/edema, hypertension, maternal anemia/low hematocrit, proteinuria/albuminuria, elevated uric acid and creatinine, pulmonary edema, elevated liver enzymes, oliguria, headache/visual disturbances/nausea/vomiting, thrombocytopenia) and fetal outcome (intrauterine death/stillbirth, death after delivery, survival) (table 3). From all the reported cases, only those publications were retained where the diagnosis of fetal hydrops associated with maternal symptoms was certain.

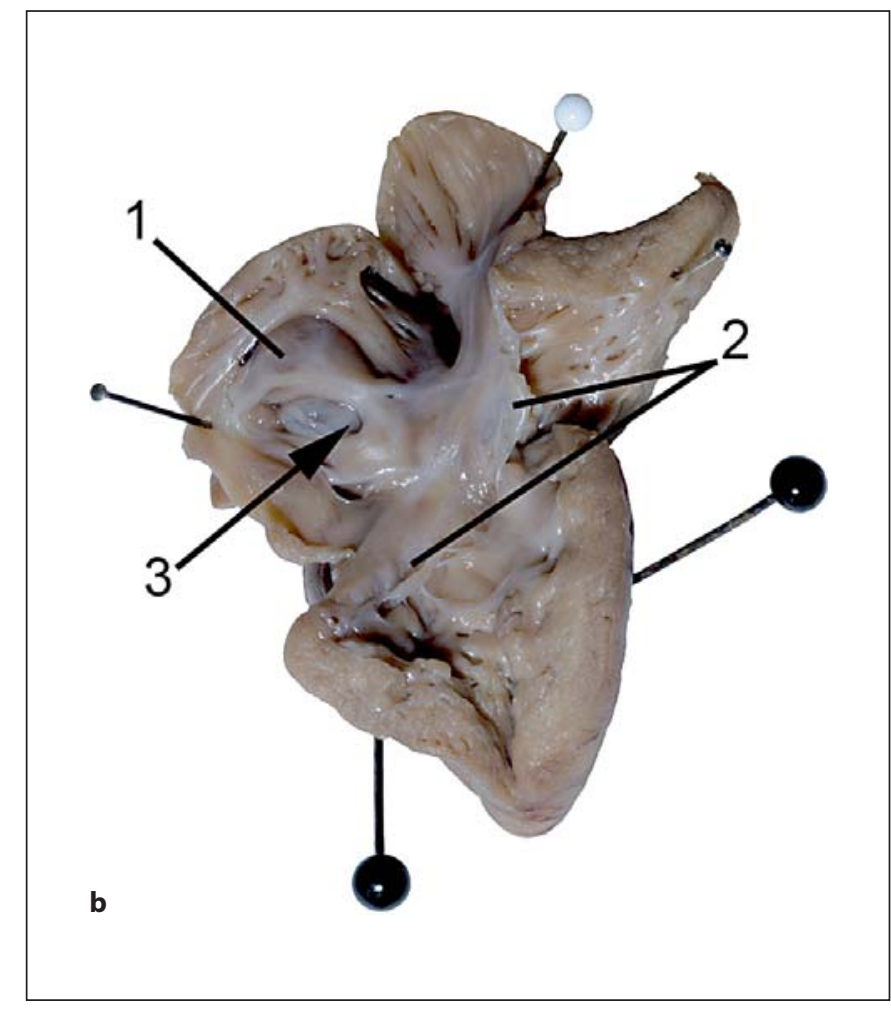

\section{Results}

After reviewing of 151 articles, 56 cases published between 1956 and 2009 met the above criteria in which cases of fetal hydrops associated with maternal symptoms were presented. Mirror syndrome could be grouped into cases associated with rhesus isoimmunization $(\mathrm{n}=15$, $28.6 \%)$, multiple pregnancies ( $\mathrm{n}=10,17.9 \%)$, viral infections $(\mathrm{n}=9,6.1 \%)$ and others including fetal malformations, fetal and placental tumors, fetal arrhythmia, etc. $(\mathrm{n}=21,37.5 \%)$ (table 3$)$. The case reports were analyzed regarding the time of diagnosis of fetal symptoms, the time when maternal symptoms appeared, the time of delivery, fetal outcome and the time of maternal reconvalescence. The time in pregnancy when maternal symptoms first occurred ranged from 16 to 34 weeks of gestation (table 1). The earliest time of diagnosis of mirror syndrome was made in a case of twin pregnancies at 16 weeks of gestation, in 4 out of 56 cases the diagnosis was made postpartum. Maternal symptoms were analyzed and could be grouped into ten (for details, see table 3). The most common maternal symptoms were weight gain and maternal edema (89.3\%), followed by elevated blood pressure (60.7\%), mild anemia and hemodilution (46.4\%), 
Table 1. Literature review with case reports of mirror syndrome

\begin{tabular}{|c|c|c|c|c|c|c|}
\hline No. & Author & $\begin{array}{l}\text { Time } \\
\text { of diag- } \\
\text { nosis }\end{array}$ & Fetal pathology & Fetal symptoms & $\begin{array}{l}\text { Time of } \\
\text { maternal } \\
\text { symptoms }\end{array}$ & Maternal symptoms \\
\hline \multicolumn{7}{|c|}{ a Rhesus isoimmunization } \\
\hline 1 & $\begin{array}{l}\text { Cohen } \\
1960[28]\end{array}$ & 28 & $\begin{array}{l}\text { rhesus } \\
\text { isoimmunization }\end{array}$ & $\begin{array}{l}\text { no ultrasound available, fetal heart sounds were not } \\
\text { present at admission; generalized edema and large and } \\
\text { pale placenta }\end{array}$ & 27 & leg edema, anemia, polyuria \\
\hline 2 & $\begin{array}{l}\text { De Silva } \\
1971[29]\end{array}$ & & $\begin{array}{l}\text { rhesus } \\
\text { isoimmunization }\end{array}$ & placenta megaly, hydrops fetalis & 27 & edema, anemia \\
\hline 3 & $\begin{array}{l}\text { Hirsch } \\
1964[30]\end{array}$ & 30 & $\begin{array}{l}\text { rhesus } \\
\text { isoimmunization }\end{array}$ & placenta megaly & 28 & edema, anemia, albuminuria \\
\hline 4 & $\begin{array}{l}\text { John } \\
1964[31]\end{array}$ & 28 & $\begin{array}{l}\text { rhesus } \\
\text { isoimmunization }\end{array}$ & hydrops fetalis (X-ray) & 26 & hypertension, edema, albuminuria \\
\hline 5 & $\begin{array}{l}\text { Keane } \\
1978[32]\end{array}$ & 29 & $\begin{array}{l}\text { rhesus } \\
\text { isoimmunization }\end{array}$ & $\begin{array}{l}\text { palpable hydramnion, fetal hydrops (X-ray), } \\
\text { placenta hydrops }\end{array}$ & 26 & edema, with 29 weeks hypertension \\
\hline 6 & $\begin{array}{l}\text { Lobato } \\
2008[33]\end{array}$ & 28 & $\begin{array}{l}\text { rhesus } \\
\text { isoimmunization }\end{array}$ & $\begin{array}{l}\text { pericardial effusion, subcutaneous edema, } \\
\text { hepatosplenomegaly, severe ascites, hydropic placenta }\end{array}$ & 28 & $\begin{array}{l}\text { hypertension, edema, proteinuria, mild anemia, } \\
\text { low hematocrit }\end{array}$ \\
\hline 7 & $\begin{array}{l}\text { Mahmood } \\
1987[34]\end{array}$ & 26 & $\begin{array}{l}\text { rhesus } \\
\text { isoimmunization }\end{array}$ & hydrops fetalis, polyhydramnion & 26 & hypertension, albuminuria, anemia \\
\hline 8 & $\begin{array}{l}\text { Nicolay } \\
1964[35]\end{array}$ & $\begin{array}{l}\text { post } \\
\text { partum }\end{array}$ & $\begin{array}{l}\text { Rhesus } \\
\text { isoimmunization }\end{array}$ & hydrops fetalis, hydropic placenta & 34 & edema, hypertension, mid proteinuria \\
\hline 9 & $\begin{array}{l}\text { Nicolay } \\
1964[35]\end{array}$ & $\begin{array}{l}\text { post } \\
\text { partum }\end{array}$ & $\begin{array}{l}\text { rhesus } \\
\text { isoimmunization }\end{array}$ & hydrops fetalis, hydropic placenta & 30 & edema, weight gain \\
\hline 10 & $\begin{array}{l}\text { Nicolay } \\
1964[35]\end{array}$ & $\begin{array}{l}\text { post } \\
\text { partum }\end{array}$ & $\begin{array}{l}\text { rhesus } \\
\text { isoimmunization }\end{array}$ & hydrops fetalis, hydropic placenta & 29 & edema, weight gain \\
\hline 11 & $\begin{array}{l}\text { O’Driscoll } \\
1956[36]\end{array}$ & 32 & $\begin{array}{l}\text { rhesus } \\
\text { isoimmunization }\end{array}$ & hydropic placenta, massive fetal ascites & 32 & $\begin{array}{l}\text { dyspnea, faintness, slight vaginal bleeding, } \\
\text { anemia, edema, mild hypertension, blebs on the } \\
\text { abdominal wall }\end{array}$ \\
\hline 12 & $\begin{array}{l}\text { Rustamov } \\
2006[37]\end{array}$ & 26 & $\begin{array}{l}\text { rhesus } \\
\text { isoimmunization }\end{array}$ & $\begin{array}{l}\text { oligohydramnion, fetal ascites, pericardial effusion, } \\
\text { fetal anemia }\end{array}$ & 26 & $\begin{array}{l}\text { moderate edema, headaches, vomiting, mild } \\
\text { hypertension, proteinuria, high urea level; } \\
26+3 \text { severe pulmonary edema, anemia, } \\
\text { thrombocytopenia }\end{array}$ \\
\hline 13 & $\begin{array}{l}\text { Van Selm } \\
1991[5]\end{array}$ & 22 & $\begin{array}{l}\text { rhesus } \\
\text { isoimmunization }\end{array}$ & severe fetal and placental hydrops & 22 & $\begin{array}{l}\text { edema, anemia, hypertension, high plasma uric } \\
\text { levels }\end{array}$ \\
\hline 14 & $\begin{array}{l}\text { Van Selm } \\
1991[5]\end{array}$ & 25 & $\begin{array}{l}\text { rhesus } \\
\text { isoimmunization }\end{array}$ & severe fetal and placental hydrops & $25+5$ & $\begin{array}{l}\text { edema, hypertension, eclamptic convulsion, } \\
\text { cerebral hemorrhagic infarction }\end{array}$ \\
\hline 15 & $\begin{array}{l}\text { Van Selm } \\
1991[5]\end{array}$ & 28 & $\begin{array}{l}\text { rhesus } \\
\text { isoimmunization }\end{array}$ & massive hydrops fetalis, anemia, placental hydrops & 28 & $\begin{array}{l}\text { edema, epigastric pain, nausea, vomiting, } \\
\text { visual disturbances }\end{array}$ \\
\hline \multicolumn{7}{|c|}{ b Twin pregnancies } \\
\hline 1 & $\begin{array}{l}\text { Heyborne } \\
2004[24]\end{array}$ & 16 & $\begin{array}{l}\text { dichorionic } \\
\text { twins }\end{array}$ & $\begin{array}{l}\text { recipient: one fetus with ascites, pleural and pericar- } \\
\text { dial effusions, generalized skin edema }\end{array}$ & 16 & $\begin{array}{l}\text { massive edema, mild thrombocytopenia, elevated } \\
\text { liver enzymes (ALT/AST) }\end{array}$ \\
\hline 2 & $\begin{array}{l}\text { Heyborne } \\
2000[23]\end{array}$ & 16 & $\begin{array}{l}\text { dichorionic } \\
\text { twins, cause for } \\
\text { fetal hydrops } \\
\text { unknown }\end{array}$ & $\begin{array}{l}\text { recipient: hydrops fetalis, ascites, pericardial effusion, } \\
\text { generalized skin edema }\end{array}$ & 16 & $\begin{array}{l}\text { rapid-onset generalized edema, elevated AST/ } \\
\text { ALT, no preeclamptic findings }\end{array}$ \\
\hline 3 & $\begin{array}{l}\text { Pirhonen } \\
2004[38]\end{array}$ & $28+1$ & $\begin{array}{l}\text { dichorionic } \\
\text { twins }\end{array}$ & $\begin{array}{l}\text { recipient: severe hydrops of the male twin, skin ede- } \\
\text { ma, ascites, pleural and pericardial effusions; terminal } \\
\text { heart insufficiency, reverse flow in the umbilical artery } \\
\text { and ductus venosus, hydropic placenta }\end{array}$ & $26+1$ & $\begin{array}{l}\text { extreme maternal edema, blood pressure increase, } \\
\text { proteinuria, elevated uric acid and creatinine, } \\
\text { high serum potassium and a low serum sodium, } \\
\text { liver enzymes were mildly elevated }\end{array}$ \\
\hline 4 & $\begin{array}{l}\text { Hayashi } \\
2006[39]\end{array}$ & $24+5$ & $\begin{array}{l}\text { monochorionic- } \\
\text { diamniotic twins } \\
\text { with TTTS }\end{array}$ & $\begin{array}{l}\text { recipient: polyhydramnion, reverse flow in the ductus } \\
\text { venosus and pulsatile umbilical venous, hypertrophic } \\
\text { cardiomegaly; prior to surgery hydrops fetalis with } \\
\text { ascites, mild skin edema, pericardiac effusion, placen- } \\
\text { ta megaly }\end{array}$ & $25+0$ & $\begin{array}{l}\text { mild edema, hemoglobin and hematocrit values } \\
\text { decreased sharply just before the operation; } \\
30 \text { min after surgery acute respiratory failure with } \\
\text { dyspnea, pulmonary edema, skin edema, oliguria, } \\
\text { acute anemia, hypoproteinemia; } \\
\text { patient stayed intubated until postoperative day } 6\end{array}$ \\
\hline 5 & $\begin{array}{l}\text { Matsubara } \\
2008[40]\end{array}$ & $23+2$ & $\begin{array}{l}\text { monochorionic, } \\
\text { triamniotic-trip- } \\
\text { lets with TTTS } \\
\text { between acardius } \\
\text { and twin A }\end{array}$ & $\begin{array}{l}\text { two living fetuses }(\mathrm{A}+\mathrm{B}) \text { with PROM, one acardius: } \\
\text { edema, placenta megaly }\end{array}$ & $23+2$ & $\begin{array}{l}\text { edema, hypertension, proteinuria, anemia, low } \\
\text { hematocrit, cardiomegaly }\end{array}$ \\
\hline 6 & $\begin{array}{l}\text { Olivella } \\
2006[41]\end{array}$ & $27+6$ & $\begin{array}{l}\text { monochorionic- } \\
\text { diamniotic twins } \\
\text { with TTTS }\end{array}$ & recipient with hydrops fetalis & $27+6$ & $\begin{array}{l}\text { hypertension, tachycardia and tachypnea, acute } \\
\text { respiratory failure and oliguria } 1 \text { day after laser } \\
\text { therapy }\end{array}$ \\
\hline
\end{tabular}




\begin{tabular}{|c|c|c|c|}
\hline Treatment & $\begin{array}{l}\text { Gestation } \\
\text { at delivery }\end{array}$ & Fetal outcome & Maternal reconvalescence \\
\hline reserpine, acetazolamide, low-salt diet & 28 & stillborn delivered by craniotomy & not reported \\
\hline $\begin{array}{l}\text { iron, vitamin } B_{12} \text {, calcium lactate, } \\
\text { hydrochlorthiazide }\end{array}$ & 30 & stillbirth & disappeared at $27+3$ weeks \\
\hline $\begin{array}{l}\text { bedrest, chlorthiazide, salt-poor diet, } \\
\text { pironolactone, ACTH, methylprednisolone, } \\
\text { maternal blood transfusion }\end{array}$ & 31 & intrauterine death & 2 weeks \\
\hline diuretics & 28 & artificial rupture of the membranes, stillbirth & not reported \\
\hline none & 29 & stillborn after induction & within $36 \mathrm{~h}$ after delivery \\
\hline $\begin{array}{l}4 \times \text { intrauterine exchange transfusion, } \\
\text { methyldopa }\end{array}$ & 32 & $\begin{array}{l}\text { after initial improvement worsening of fetal symptoms, } \\
\text { cesarean section, discharged } 46 \text { days after birth }\end{array}$ & $\begin{array}{l}\text { shortly after the forth intrauterine infu- } \\
\text { sion ( } 31 \text { weeks of gestation) }\end{array}$ \\
\hline none & 27 & PROM, fetus died $30 \mathrm{~min}$ after delivery & $\begin{array}{l}\text { febrile infection, normalization } 10 \text { days } \\
\text { after delivery }\end{array}$ \\
\hline various diuretics & $35+2$ & died $30 \mathrm{~min}$ after delivery & within $72 \mathrm{~h}$ after delivery \\
\hline various diuretics & 33 & premature labor, paracentesis to effect delivery & within $72 \mathrm{~h}$ after delivery \\
\hline not reported & 30 & spontaneous labor, died within minutes after delivery & not reported \\
\hline none & not reported & spontaneous delivery, not reported & $48 \mathrm{~h}$ after delivery \\
\hline intrauterine exchange transfusion & $26+3$ & died $1 \mathrm{~h}$ after delivery & improved rapidly after delivery \\
\hline none & 27 & intrauterine death & $12 \mathrm{~h}$ after deliver \\
\hline intrauterine exchange transfusion & 27 & intrauterine death & 1 week after eclamptic convulsion \\
\hline intrauterine exchange transfusion & $28+4$ & intrauterine death & 2 days after delivery \\
\hline $\begin{array}{l}\text { dexamethasone, selective fetocide of the } \\
\text { affected twin }\end{array}$ & term & healthy & $1-2$ weeks after delivery \\
\hline $\begin{array}{l}\text { dexamethasone, selective fetocide of the } \\
\text { affected twin }\end{array}$ & term & healthy & 1-2 weeks after delivery \\
\hline not reported & $29+4$ & $\begin{array}{l}\text { stillbirth of hydropic twin, } 31+3 \text { weeks cesarean section } \\
\text { due to preterm labor, female fetus healthy }\end{array}$ & $\begin{array}{l}\text { normalization of blood pressure within } \\
24 \mathrm{~h} \text { after hydropic twin died, after } 3 \\
\text { weeks full remission }\end{array}$ \\
\hline $\begin{array}{l}2 \times \text { amnion fluid drainage in } 3 \text { days, laser } \\
\text { ablation therapy with } 27 \text { weeks }\end{array}$ & $25+1$ & $\begin{array}{l}\text { stillbirth of recipient twin, ascites of donor twin on } \\
\text { postoperative day } 1 \text {, delivered on postoperative day } 6 \text {, } \\
\text { recipient died on postoperative day } 13 \text { due to severe } \\
\text { acidemia and sepsis }\end{array}$ & shortly after delivery \\
\hline not reported & $23+5$ & emergency cesarean section due to cord prolapse & 3 days after delivery \\
\hline successful laser ablation therapy & not reported & not reported & not reported \\
\hline
\end{tabular}


Table 1 (continued)

\begin{tabular}{|c|c|c|c|c|c|c|}
\hline No. & Author & $\begin{array}{l}\text { Time } \\
\text { of diag- } \\
\text { nosis }\end{array}$ & Fetal pathology & Fetal symptoms & $\begin{array}{l}\text { Time of } \\
\text { maternal } \\
\text { symptoms }\end{array}$ & Maternal symptoms \\
\hline 7 & $\begin{array}{l}\text { Olivella } \\
2006[41]\end{array}$ & $19+3$ & $\begin{array}{l}\text { monochorionic- } \\
\text { diamniotic } \\
\text { twins with TTTS }\end{array}$ & recipient: severe polyhydramnion & $19+3$ & hypertension, elevated liver enzymes, anemia \\
\hline 8 & $\begin{array}{l}\text { Kumar } \\
2007[8]\end{array}$ & 26 & $\begin{array}{l}\text { monochorionic- } \\
\text { diamniotic twins } \\
\text { with TTTS }\end{array}$ & $\begin{array}{l}\text { recipient: polyhydramnion, enlarged heart, poorly } \\
\text { contracting, ascites; donor: oligohydramnion }\end{array}$ & 27 & hypertension, generalized edema \\
\hline 9 & $\begin{array}{l}\text { Chang } \\
2007[42]\end{array}$ & 19 & $\begin{array}{l}\text { dichorionic- } \\
\text { triplets with } \\
\text { TTTS }\end{array}$ & $\begin{array}{l}\text { one set of female twins with poly-/oligohydramnion } \\
\text { sequence, male fetus with a normal amount of amni- } \\
\text { otic fluid, recipient twin reverse A wave of the ductus } \\
\text { venosus and pulsation of the umbilical vein }\end{array}$ & 19 & $\begin{array}{l}\text { severe edema, hypertension, mild anemia, high } \\
\text { uric acid, high LDH level }\end{array}$ \\
\hline 10 & $\begin{array}{l}\text { Matsubara } \\
2008[43]\end{array}$ & $21+3$ & $\begin{array}{l}\text { monochorionic- } \\
\text { twins with FTTS }\end{array}$ & $\begin{array}{l}\text { recipient: polyhydramnion, hydrops fetalis, skin } \\
\text { edema, ascites, pleural effusion, hypertrophic cardio- } \\
\text { megaly }\end{array}$ & $21+3$ & $\begin{array}{l}\text { weight gain, anemia, low albuminemia, elevated } \\
\text { hCG; initial worsening of maternal symptoms } \\
4 \text { days after surgery }\end{array}$ \\
\hline
\end{tabular}

c Viral infections

1 Ambrosini 26+5 Coxsackie B virus hydrops fetalis, ascites, pericardial effusion, polyhy2008 [15] dramnion, skin and placenta edema, increased middle cerebral artery peak systolic velocity, fetal anemia

\begin{tabular}{llll}
\hline 4 & Brochot & 20 & parvovirus B19 \\
$2006[13]$ & &
\end{tabular}

audible heart tones; obduction: hydrops fetalis, pericardial effusion, ascites, hepato- and splenomegaly, edematous placenta

ascites, pleural effusions, placental edema, fetal skin edema pleural effusion, hepatosplenomegaly, cardiomegaly, pulmonary hypoplasia, pathologic CTG

hydrops fetalis and IUGR,

fetal anemia

\begin{tabular}{llll}
\hline 2 & $\begin{array}{l}\text { Quagliarello } \\
1978[44]\end{array}$ & & cytomegalovirus \\
& & & \\
\hline 3 & $\begin{array}{l}\text { Rana } \\
\text { 2007 [19] }\end{array}$ & $29+4$ & cytomegalovirus \\
& & \\
\hline
\end{tabular}

27 dilution anemia; after transfusion increased edema, oliguria, anemia; $27+3$ weeks peripartum cardiomyopathy: hypotension, cyanosis, generalized edema edema, hypertension

30 edema, hypertension

$29+4$ worsening leg swelling over 1 week, hypertension, proteinuria

25 moderate hypertension, elevated liver enzymes, hyperuricemia, $48 \mathrm{~h}$ later edema, hemodilution, epigastric pain, pulmonary edema, pleura effusions

\begin{tabular}{|c|c|c|c|c|c|c|}
\hline 5 & $\begin{array}{l}\text { Duthie } \\
1995[45]\end{array}$ & 25 & parvovirus B19 & $\begin{array}{l}\text { hydrops fetalis, oligohydramnion, hydropic placenta, } \\
\text { fetal anemia }\end{array}$ & 25 & $\begin{array}{l}\text { edema, proteinuria, hypertension, moderate } \\
\text { thrombocytopenia }\end{array}$ \\
\hline 6 & $\begin{array}{l}\text { Goeden } \\
2005[7]\end{array}$ & $21+5$ & parvovirus B19 & $\begin{array}{l}\text { hydrops fetalis, scalp edema, pleural and pericardial } \\
\text { effusions, ascites, thickened placenta }\end{array}$ & $23+4$ & $\begin{array}{l}\text { edema, anemia, hypertension, proteinuria, } \\
\text { hyperuricemia }\end{array}$ \\
\hline 7 & $\begin{array}{l}\text { Proust } \\
2006[46]\end{array}$ & 25 & parvovirus B19 & hydrops fetalis, anasarca, placenta hypertrophy & 25 & edema, proteinuria, hypertension \\
\hline 8 & $\begin{array}{l}\text { Proust } \\
2006[46]\end{array}$ & 22 & parvovirus B19 & hydrops fetalis, anemia, placenta hypertrophy & $24+6$ & $\begin{array}{l}\text { edema, proteinuria, pruritus, hyperuricemia, } \\
\text { elevated liver enzymes }\end{array}$ \\
\hline 9 & $\begin{array}{l}\text { Ville } \\
1995[14]\end{array}$ & 25 & parvovirus B19 & $\begin{array}{l}\text { fetal and placental hydrops, ascites, subcutaneous } \\
\text { edema, heart dilatation, pericardial effusion }\end{array}$ & 25 & $\begin{array}{l}\text { chest pain, headache, leg and hand edema, mild } \\
\text { hypertony; right pleural effusion and bilateral } \\
\text { interstitial syndrome; hyponatremia, hypoosmo- } \\
\text { lality and secondary hyperaldosteronism }\end{array}$ \\
\hline
\end{tabular}

d Tumors, congenital anomalies and others

1 Ordorica 27/33 aneurysm of 1990 [9] vein of Galen

27 weeks: hydrops fetalis, vein

30 edema, mildly proteinuria, pulmonary edema

of Galeni malformation, polyhydramnion;

33 weeks: ascites, cardiomegaly, placenta megaly

2 Mizrahi- 29 aortic stenosis hydrops fetalis, pericardial effusion, ascites, aortic stenosis, dilated left ventricle, polyhydramnion, 2006 [47] $\quad 31 \quad$ placenta megaly

\begin{tabular}{llll}
\hline & Wurm & 31 & $\begin{array}{l}\text { congenital disor- } \\
\text { ders of glycosyl- }\end{array}$
\end{tabular} ation type Ia

4 Paternoster 28+4 cystic hygroma at 2006 [49] the fetal neck

hydrothorax, subcutaneous edema, placental edema, 30 polyhydramnion, borderline ventriculomegaly, pleural effusion, edematous umbilical cord, absent or reversed end-diastolic flow, increased pulsatility index in middle cerebral artery

$5 \quad$ Valsky $\quad 25+6 \quad$ Diamond-Black-
2007 [50] fan anemia hydrops fetalis, severe ascites, pericardial effusion, scalp edema, pronounced cardiomegaly myocardial hypertrophy, biventricular dilatation with tricuspid and mitral regurgitation, dilatation of the vena cava, reversed flow in the ductus venosus, oligohydramnion, fetal anemia; at $26+6$ weeks severe placental edema with dyspnea, next week renal insufficiency, hypertension

30+4 after surgery, pulmonary edema, impaired hepatic function, low thyroid-stimulating hormone, high chorionic gonadotropin

31 maternal edema, mild oliguria

$30 \quad$ edema of hands and face, anemia proteinuria, decreased hematocrit elevated liver enzymes, uric acid was elevated rapidly increasing maternal edema, hypertension, 


\begin{tabular}{|c|c|c|c|}
\hline Treatment & $\begin{array}{l}\text { Gestation } \\
\text { at delivery }\end{array}$ & Fetal outcome & Maternal reconvalescence \\
\hline none & not reported & termination of pregnancy & not reported \\
\hline $\begin{array}{l}\text { successful laser ablation therapy with } \\
27 \text { weeks; donor developed ascites and hy- } \\
\text { drops afterwards }\end{array}$ & 27 & $\begin{array}{l}\text { donor with progressive ascites after cesarean section, an- } \\
\text { uric, developed RDS and died } 1 \text { week after delivery }\end{array}$ & shortly after cesarean section \\
\hline laser therapy was not possible & $19+1$ & $\begin{array}{l}\text { termination of recipient twin with electrocoagulation } \\
\text { of the umbilical cord; postoperative day } 2 \text { donor twin died } \\
\text { as well; miscarriage with } 21 \text { weeks due to vaginal bleeding }\end{array}$ & 1-2 days after termination \\
\hline fetoscopic laser photocoagulation & 37 & cesarean section & 10 days after surgery \\
\hline intrauterine blood transfusion & $27+3$ & emergency cesarean section, healthy & 17 days after delivery \\
\hline not reported & $30+2$ & intrauterine death, spontaneous delivery with $31+2$ weeks & not reported \\
\hline not reported & $29+5$ & $\begin{array}{l}\text { emergency cesarean section, neonate had severe anemia } \\
\text { and lived } 1 \mathrm{~h} \text { for the duration of resuscitation }\end{array}$ & not reported \\
\hline $\begin{array}{l}\text { nicardipine followed by labetalol and } \\
\text { diuretics, } 2 \text { intrauterine exchange trans- } \\
\text { fusions; intensive care unit with positive } \\
\text { pressure ventilation }\end{array}$ & 36 & $\begin{array}{l}\text { MRT at } 32 \text { weeks revealed severe fetal cerebral abnormali- } \\
\text { ties, fetocide and induction of labor at } 36 \text { weeks }\end{array}$ & shortly after delivery \\
\hline $\begin{array}{l}\text { intrauterine transfusion, day } 7 \text { fetal ascites } \\
\text { disappeared }\end{array}$ & 37 & spontaneous delivery, healthy newborn & few weeks after delivery \\
\hline $\begin{array}{l}\text { lung maturation, intrauterine exchange } \\
\text { transfusion unsuccessful }\end{array}$ & $36+6$ & healthy & $\begin{array}{l}28+4 \text { weeks of gestation spontaneous } \\
\text { complete remission of maternal and fetal } \\
\text { symptoms }\end{array}$ \\
\hline none & 25 & intrauterine death & not reported \\
\hline intrauterine transfusion & 25 & intrauterine death & few days after delivery \\
\hline water depletion, $20 \mathrm{~g}$ albumin & $25+3$ & induction with prostaglandins after intrauterine death & $\begin{array}{l}18 \mathrm{~h} \text { after intrauterine death maternal } \\
\text { RDS, } \\
\text { diuretics, several pleural drainages, } \\
\text { discharged } 10 \text { days after delivery }\end{array}$ \\
\hline
\end{tabular}

digoxin, furosemide

digoxin, fetal aortic valve dilatation

not reported

2 pleuroamniotic shunts with $29+4$,

disappearance of hydrothorax
34

$30+6$

36 cesarean section, neonate died shortly after delivery of car- 5 days after delivery diovascular collapse

cesarean section, balloon dilatation, died of fungal sepsis $13 \quad 7$ days after delivery days after birth

not reported

$$
\text { cesarean section }
$$

$30+3$

cesarean section because of pathologic CTG

6 days after delivery

\begin{tabular}{|c|c|c|c|}
\hline $\begin{array}{l}2 \times \text { intrauterine blood transfusion at } 26 / 28 \\
\text { weeks; magnesium sulfate }\end{array}$ & $34+1$ & $\begin{array}{l}\text { cesarean section, Diamond-Blackfan anemia as postnatal } \\
\text { diagnosis }\end{array}$ & $\begin{array}{l}30 \text { weeks of gestation symptoms com- } \\
\text { pletely disappeared }\end{array}$ \\
\hline
\end{tabular}


Table 1 (continued)

\begin{tabular}{|c|c|c|c|c|c|c|}
\hline No. & Author & $\begin{array}{l}\text { Time } \\
\text { of diag- } \\
\text { nosis }\end{array}$ & Fetal pathology & Fetal symptoms & $\begin{array}{l}\text { Time of } \\
\text { maternal } \\
\text { symptoms }\end{array}$ & Maternal symptoms \\
\hline 6 & $\begin{array}{l}\text { Carbillon } \\
1997[6]\end{array}$ & $30+3$ & $\begin{array}{l}\text { Ebstein's } \\
\text { anomaly }\end{array}$ & $\begin{array}{l}\text { hydrops fetalis, pleural effusion, massive tricuspid } \\
\text { reverse flow, pulmonary atresia, placental edema, } \\
\text { polyhydramnion }\end{array}$ & $30+4$ & edema, anemia, thrombocytopenia \\
\hline 7 & $\begin{array}{l}\text { Leung } \\
2006[27]\end{array}$ & 27 & $\begin{array}{l}\text { Ebstein's } \\
\text { anomaly }\end{array}$ & $\begin{array}{l}\text { cardiomegaly, ascites, subcutaneous edema, peri- } \\
\text { cardial effusion, polyhydramnion, placental edema }\end{array}$ & 29 & $\begin{array}{l}\text { edematous, right upper quadrant abdominal pain } \\
\text { and palpitations, sinus tachycardia, elevated liver } \\
\text { enzymes, borderline hypothyroidism, oliguria, } \\
\text { increased creatinine and urate level, anemia, } \\
\text { during labor induction impending eclampsia }\end{array}$ \\
\hline 8 & $\begin{array}{l}\text { Livingston } \\
2007[22]\end{array}$ & $29+2$ & $\begin{array}{l}\text { endodermal } \\
\text { sinus tumor } \\
\text { of the } \\
\text { sacrococcygeal } \\
\text { region }\end{array}$ & $\begin{array}{l}\text { heterogeneous mass protruding in the sacrococcygeal } \\
\text { area, fetal abdominal urinary ascites, skin edema, } \\
\text { placenta megaly, oligohydramnion, hydroureter, } \\
\text { hydronephrosis, evidence of bladder rupture }\end{array}$ & $29+2$ & $\begin{array}{l}\text { edema, shortness of breath, chest pain, palpita- } \\
\text { tions, blurred vision, hypertension, anemia, } \\
\text { hyperuricemia; } 30+1 \text { weeks dyspnea, tachycardia }\end{array}$ \\
\hline 9 & $\begin{array}{l}\text { Allen } \\
2007[51]\end{array}$ & $27+6$ & $\begin{array}{l}\text { fetal renal } \\
\text { neuroblastoma, } \\
\text { metastatic } \\
\text { to the placenta }\end{array}$ & $\begin{array}{l}\text { hydrops fetalis, mass within the left kidney, scalp } \\
\text { edema, placenta megaly }\end{array}$ & $28+6$ & edema, mild hypertension, oliguria \\
\hline 10 & $\begin{array}{l}\text { Dorman } \\
1995[11]\end{array}$ & $>19$ & $\begin{array}{l}\text { placental } \\
\text { chorangioma }\end{array}$ & hydropic fetus, thickened placenta & $>19$ & $\begin{array}{l}\text { mild frontal headache, slight nausea, marked } \\
\text { peripheral edema, hypertension, proteinuria }\end{array}$ \\
\hline 11 & $\begin{array}{l}\text { Gherman } \\
1998[52]\end{array}$ & 25 & $\begin{array}{l}\text { placental } \\
\text { chorangioma }\end{array}$ & $\begin{array}{l}23 \text { weeks: polyhydramnion; } 25 \text { weeks: placental mass, } \\
\text { hydrops fetalis }\end{array}$ & 25 & $\begin{array}{l}\text { acute onset lower extremity edema, hyper- } \\
\text { uricemia, generalized pruritus, dyspnea, hemo- } \\
\text { dilutional anemia, pre-term labor; markedly } \\
\text { elevated human chorionic gonadotrophin }\end{array}$ \\
\hline 12 & $\begin{array}{l}\text { Galimberti } \\
2000[53]\end{array}$ & 26 & $\begin{array}{l}\text { placental choran- } \\
\text { gioma, cardiac } \\
\text { failure secondary } \\
\text { to shunting } \\
\text { through the } \\
\text { placental tumor }\end{array}$ & $\begin{array}{l}\text { subcutaneous edema, ascites, pleura effusion, } \\
\text { polyhydramnion, suspicious mass adjacent to the } \\
\text { placenta }\end{array}$ & 26 & $\begin{array}{l}\text { edema, mild hypertension, proteinuria, hyper- } \\
\text { reflexia, anemia, mildly elevated urate }\end{array}$ \\
\hline 13 & $\begin{array}{l}\text { Deurloo } \\
2003[54]\end{array}$ & 29 & $\begin{array}{l}\text { primary chylo- } \\
\text { thorax with sec- } \\
\text { ondary hydrops } \\
\text { and polyhydram- } \\
\text { nion due to car- } \\
\text { diac and esopha- } \\
\text { geal compression }\end{array}$ & $\begin{array}{l}\text { severe polyhydramnion, hydrops fetalis, bilateral } \\
\text { hydrothorax, increased placental thickness }\end{array}$ & 30 & $\begin{array}{l}\text { abdominal overdistension, pain, headache, visual } \\
\text { disturbances, back pain, massive edema, very } \\
\text { active patellar reflexes, hypertensive, proteinuria, } \\
\text { slightly raised liver enzymes, anemia, mild albu- } \\
\text { minuria }\end{array}$ \\
\hline 14 & $\begin{array}{l}\text { Finamore } \\
2007[10]\end{array}$ & 29 & $\begin{array}{l}\text { sacrococcygeal } \\
\text { teratoma }\end{array}$ & $\begin{array}{l}29 \text { weeks increased cerebral flow; } 29+5 \text { weeks enlarged } \\
\text { heart, thickened placenta, increased liver length, mild } \\
\text { polyhydramnion }\end{array}$ & 30 & $\begin{array}{l}\text { pruritic rash, edema, hypertension, epigastric } \\
\text { pain, proteinuria, anemia and thrombocytopenia }\end{array}$ \\
\hline 15 & $\begin{array}{l}\text { Ibele } \\
2008[55]\end{array}$ & $25+3$ & $\begin{array}{l}\text { sacrococcygeal } \\
\text { teratoma }\end{array}$ & $\begin{array}{l}\text { mild pericardial effusion, } 27 \text { weeks: placenta megaly, } \\
\text { polyhydramnion, scalp edema, ascites, pericardial and } \\
\text { pleural effusions }\end{array}$ & $27+2$ & $\begin{array}{l}\text { hypertension, edema, highly elevated liver en- } \\
\text { zymes }\end{array}$ \\
\hline 16 & $\begin{array}{l}\text { Midgley } \\
2000[12]\end{array}$ & 27 & $\begin{array}{l}\text { supraventricular } \\
\text { tachycardia }\end{array}$ & pericardial effusion, scalp edema, ascites & 27 & $\begin{array}{l}\text { headaches, visual disturbances, hypertension, } \\
\text { proteinuric, raised uric acid }\end{array}$ \\
\hline 17 & $\begin{array}{l}\text { John } \\
1964[31]\end{array}$ & 30 & unknown & hydrops fetalis (X-ray); placenta megaly & 28 & edema, with 30 weeks hypertension, proteinuria \\
\hline 18 & $\begin{array}{l}\text { Kaiser } \\
1971[4]\end{array}$ & $\begin{array}{l}\text { after } \\
\text { delivery }\end{array}$ & unknown & $\begin{array}{l}\text { hydrops fetalis, ascites, pericardial effusion, large and } \\
\text { edematous placenta }\end{array}$ & 28 & $\begin{array}{l}\text { edema, mild anemia, later dyspnea, oliguria, mild } \\
\text { proteinuria, hypertension }\end{array}$ \\
\hline 19 & $\begin{array}{l}\text { Nakamura } \\
2002[56]\end{array}$ & 23 & unknown & $\begin{array}{l}\text { subcutaneous edema, massive ascites, hydrothorax, } \\
\text { single heart ventricle, common atrioventricular canal, } \\
\text { enlarged placenta }\end{array}$ & 23 & $\begin{array}{l}\text { hydrothorax, generalized edema, proteinuria, } \\
\text { mild hypertension }\end{array}$ \\
\hline 20 & $\begin{array}{l}\text { Vidaeff } \\
2002[57]\end{array}$ & 31 & unknown & hydramnion and fetal ascites & 31 & massive edema, anemia \\
\hline 21 & $\begin{array}{l}\text { Vidaeff } \\
2003[58]\end{array}$ & 32 & unknown & $\begin{array}{l}\text { hydramnion, scalp edema, pleural effusions, mild } \\
\text { ascites, placenta megaly }\end{array}$ & 32 & edema \\
\hline
\end{tabular}

TTTS $=$ Twin-to-twin transfusion syndrome 


\begin{tabular}{|c|c|c|c|}
\hline Treatment & $\begin{array}{l}\text { Gestation } \\
\text { at delivery }\end{array}$ & Fetal outcome & Maternal reconvalescence \\
\hline bedrest & $31+3$ & stillbirth & 4 days after delivery \\
\hline
\end{tabular}

\begin{tabular}{|c|c|c|c|}
\hline $\begin{array}{l}\text { furosemide, fluid restriction; } 30+2 \text { weeks } \\
\text { fetal peritoneal-amniotic shunt with fast } \\
\text { removal of fetal and maternal symptoms }\end{array}$ & $34+4$ & $\begin{array}{l}\text { cesarean section, successful surgery and removal of } \\
\text { the sacrococcygeal mass }\end{array}$ & 3 days after shunting \\
\hline not reported & 29 & $\begin{array}{l}\text { pulmonary hypertension, sepsis, and renal failure, } \\
\text { death on day } 4 \text { of life }\end{array}$ & endometritis postpartum \\
\hline methyldopamine & $19+$ & stillborn hydropic fetus & 2 days after delivery \\
\hline $\begin{array}{l}\text { magnesium sulfate tocolysis, therapeutic } \\
\text { amniocentesis }\end{array}$ & 26 & secondary cesarean section, died 2 days later & $\begin{array}{l}\text { after delivery preeclampsia-like syndrome } \\
\text { with oliguria and pulmonary effusions, } \\
3 \text { weeks later complete remission }\end{array}$ \\
\hline none & 26 & $\begin{array}{l}\text { emergency cesarean section following abnormal } \\
\text { cardiotocographic trace, died after unsuccessful } \\
\text { resuscitation }\end{array}$ & 6 days after delivery \\
\hline $\begin{array}{l}\text { ultrasound-guided thoracocentesis, within } \\
24 \text { h recurrence of hydrothorax and polyhy- } \\
\text { dramnion; worsening fetal hydrops led to } \\
\text { the decision for thoracoamniotic shunting; } \\
\text { two silastic 'Rodeck' pigtail shunts were suc- } \\
\text { cessfully inserted on both sides of the thorax }\end{array}$ & $30+1$ & $\begin{array}{l}\text { neonate required intubation successful postnatal } \\
\text { drainage }\end{array}$ & 4 days after delivery \\
\hline intrauterine blood transfusion & 30 & cesarean section, successful surgery after delivery & shortly after delivery \\
\hline not reported & $27+5$ & $\begin{array}{l}\text { emergency cesarean section, discharge at day } 60 \text { after fetal } \\
\text { surgery }\end{array}$ & not reported \\
\hline felcainide & $40+1$ & healthy, no treatment after delivery & $\begin{array}{l}1 \text { week after flecainide treatment and } \\
\text { resolvement of fetal SVTs }\end{array}$ \\
\hline not reported & 30 & amniotomy, healthy & not reported \\
\hline diuretics, digitalis, phenobarbital & 32 & cesarean section, neonate died shortly after delivery & few days after delivery \\
\hline not reported & $25+0$ & intrauterine death with $24+6$ weeks, induction of labor & $\begin{array}{l}\text { after } 6 \text { days, peripartum cardiomyopathy } \\
\text { last for } 23 \text { days after delivery }\end{array}$ \\
\hline not reported & $31+3$ & healthy, ascites resolved rapidly & few days after delivery \\
\hline not reported & $32+1$ & $\begin{array}{l}\text { cesarean section due to maternal cerebral cavernous } \\
\text { hemangiomas, fetus died } 24 \mathrm{~h} \text { later due to pulmonary } \\
\text { hypoplasia }\end{array}$ & $\begin{array}{l}48 \mathrm{~h} \text { after delivery breast enlargement, } \\
\text { next day multiple blisters, superficial ne- } \\
\text { crosis, skin breakdown; in less than } 24 \mathrm{~h} \text {, } \\
\text { fever, acute respiratory distress syn- } \\
\text { drome, and acute renal failure, intubated } \\
\text { and transferred to the intensive care unit, } \\
\text { after breast reduction fast recovery }\end{array}$ \\
\hline
\end{tabular}


Table 2. Blood count and plasmatic ionogram

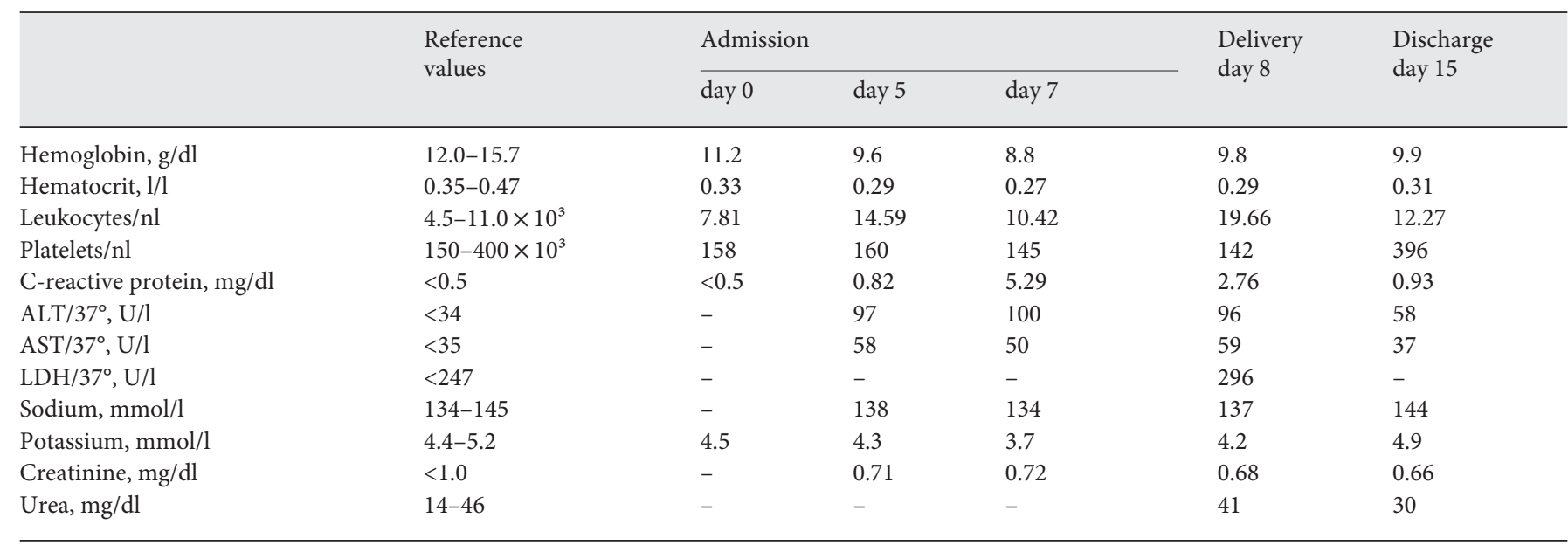

Table 3. Mirror syndrome - analyses of the literature review

\begin{tabular}{|c|c|c|c|c|c|c|c|c|c|c|c|c|c|}
\hline \multirow[t]{2}{*}{ Fetal pathology } & \multirow[t]{2}{*}{ A } & \multirow[t]{2}{*}{ B } & \multirow[t]{2}{*}{$\mathrm{C}$} & \multicolumn{10}{|c|}{ Maternal symptoms } \\
\hline & & & & $\mathrm{D}$ & $\mathrm{E}$ & $\mathrm{F}$ & G & $\mathrm{H}$ & I & $\mathrm{J}$ & $\mathrm{K}$ & $\mathrm{L}$ & M \\
\hline $\begin{array}{l}\text { Rhesus } \\
\text { isoimmunization }\end{array}$ & $\begin{array}{l}15 \\
(28.6)\end{array}$ & $\begin{array}{l}27.4 \pm \\
2.6^{1}\end{array}$ & $\begin{array}{l}27.7 \pm \\
2.8\end{array}$ & $\begin{array}{l}14 \\
(93.3)\end{array}$ & $\begin{array}{l}9 \\
(60)\end{array}$ & $\begin{array}{l}8 \\
(53.3)\end{array}$ & $\begin{array}{l}6 \\
(40)\end{array}$ & $\begin{array}{l}3 \\
(20.0)\end{array}$ & $\begin{array}{l}2 \\
(13.4)\end{array}$ & - & - & $\begin{array}{l}2 \\
(13.4)\end{array}$ & $\begin{array}{l}1 \\
(6.7)\end{array}$ \\
\hline Multiple pregnancies & $\begin{array}{l}10 \\
(17.9)\end{array}$ & $\begin{array}{l}22.5 \pm \\
4.7\end{array}$ & $\begin{array}{l}22.4 \pm \\
4.5\end{array}$ & \multirow[t]{3}{*}{$\begin{array}{l}8 \\
(80)\end{array}$} & \multirow[t]{3}{*}{$\begin{array}{l}6 \\
(60)\end{array}$} & \multirow[t]{3}{*}{$\begin{array}{l}5 \\
(50)\end{array}$} & \multirow[t]{3}{*}{$\begin{array}{l}2 \\
(20)\end{array}$} & \multirow[t]{3}{*}{$\begin{array}{l}2 \\
(20)\end{array}$} & \multirow[t]{3}{*}{$\begin{array}{l}2 \\
(20)\end{array}$} & \multirow[t]{3}{*}{$\begin{array}{l}5 \\
(50)\end{array}$} & \multirow[t]{3}{*}{$\begin{array}{l}3 \\
(30)\end{array}$} & \multirow[t]{3}{*}{-} & \multirow[t]{3}{*}{$\begin{array}{l}1 \\
(10)\end{array}$} \\
\hline TTTS & $\begin{array}{l}7 \\
(70)\end{array}$ & $\begin{array}{l}23.4 \pm \\
3.3\end{array}$ & $\begin{array}{l}23.6 \pm \\
4.4\end{array}$ & & & & & & & & & & \\
\hline Unknown & $\begin{array}{l}3 \\
(30)\end{array}$ & $\begin{array}{l}20.3 \pm \\
7.5\end{array}$ & $\begin{array}{l}19.7 \pm \\
6.4\end{array}$ & & & & & & & & & & \\
\hline Viral infections & $\begin{array}{l}9 \\
(6.1)\end{array}$ & $\begin{array}{l}25.1 \pm \\
3.5\end{array}$ & $\begin{array}{l}26.2 \pm \\
2.3\end{array}$ & \multirow[t]{4}{*}{$\begin{array}{l}9 \\
(100)\end{array}$} & \multirow[t]{4}{*}{$\begin{array}{l}7 \\
(77.8)\end{array}$} & \multirow[t]{4}{*}{$\begin{array}{l}3 \\
(33.3)\end{array}$} & \multirow[t]{4}{*}{$\begin{array}{l}5 \\
(55.6)\end{array}$} & \multirow[t]{4}{*}{$\begin{array}{l}3 \\
(33.3)\end{array}$} & \multirow[t]{4}{*}{$\begin{array}{l}2 \\
(22.2)\end{array}$} & \multirow[t]{4}{*}{$\begin{array}{l}2 \\
(22.2)\end{array}$} & \multirow[t]{4}{*}{$\begin{array}{l}1 \\
(11.1)\end{array}$} & \multirow[t]{4}{*}{$\begin{array}{l}1 \\
(11.1)\end{array}$} & \multirow[t]{4}{*}{$\begin{array}{l}1 \\
(11.1)\end{array}$} \\
\hline Parvovirus B19 & $\begin{array}{l}6 \\
(66.7)\end{array}$ & $\begin{array}{l}23.2 \pm \\
2.1\end{array}$ & $\begin{array}{l}24.8 \pm \\
0.4\end{array}$ & & & & & & & & & & \\
\hline CMV & $\begin{array}{l}2 \\
(22.2)\end{array}$ & $\begin{array}{l}30 \pm \\
0\end{array}$ & 30.0 & & & & & & & & & & \\
\hline Coxsackie B & $\begin{array}{l}1 \\
(11.1)\end{array}$ & 27 & 27 & & & & & & & & & & \\
\hline Others & $\begin{array}{l}21 \\
(37.5)\end{array}$ & $\begin{array}{l}27.8 \pm \\
3.0\end{array}$ & $\begin{array}{l}28.4 \pm \\
3.3\end{array}$ & \multirow[t]{6}{*}{$\begin{array}{l}19 \\
(90.5)\end{array}$} & \multirow[t]{6}{*}{$\begin{array}{l}12 \\
(57.1)\end{array}$} & \multirow[t]{6}{*}{$\begin{array}{l}10 \\
(47.6)\end{array}$} & \multirow[t]{6}{*}{$\begin{array}{l}11 \\
(52.4)\end{array}$} & \multirow[t]{6}{*}{$\begin{array}{l}6 \\
(28.6)\end{array}$} & \multirow[t]{6}{*}{$\begin{array}{l}6 \\
(28.6)\end{array}$} & $\begin{array}{l}4 \\
(19.0)\end{array}$ & $\begin{array}{l}5 \\
(23.8)\end{array}$ & $\begin{array}{l}5 \\
(23.8)\end{array}$ & $\begin{array}{l}1 \\
(4.8)\end{array}$ \\
\hline $\begin{array}{l}\text { Malformation, } \\
\text { arrhythmia }\end{array}$ & $\begin{array}{l}6 \\
(28.6)\end{array}$ & $\begin{array}{l}28.3 \pm \\
1.6\end{array}$ & $\begin{array}{l}29.7 \pm \\
1.5\end{array}$ & & & & & & & & & & \\
\hline Fetal tumor & $\begin{array}{l}5 \\
(23.8)\end{array}$ & $\begin{array}{l}28.4 \pm \\
1.5\end{array}$ & $\begin{array}{l}29.4 \pm \\
0.9\end{array}$ & & & & & & & & & & \\
\hline Placental tumor & $\begin{array}{l}3 \\
(14.3)\end{array}$ & $\begin{array}{l}23.7 \pm \\
3.2\end{array}$ & $\begin{array}{l}23.7 \pm \\
3.2\end{array}$ & & & & & & & & & & \\
\hline $\begin{array}{l}\text { Metabolic } \\
\text { disorders }\end{array}$ & $\begin{array}{l}2 \\
(9.5)\end{array}$ & $\begin{array}{l}29 \pm \\
4.2\end{array}$ & $\begin{array}{l}28.4 \pm \\
3.5\end{array}$ & & & & & & & & & & \\
\hline Unknown & $\begin{array}{l}5 \\
(23.8)\end{array}$ & $\begin{array}{l}29 \pm \\
4.1^{2}\end{array}$ & & & & & & & & & & & \\
\hline $\begin{array}{l}\text { Values in parenth } \\
56 \text { ); } \mathrm{B}=\text { time of diagn } \\
\text { symptoms (weeks of } \mathrm{g} \\
\text { tension; } \mathrm{F}=\text { anemia, } \\
\text { elevated uric acid and }\end{array}$ & $\begin{array}{l}\text { re percer } \\
\text { weeks of } \\
\text { on } \pm S D \\
\text { ematocri } \\
\text { tinine; I }\end{array}$ & $\begin{array}{l}\text { ges. } A= \\
\text { tation } \pm \\
D=\text { weig } \\
G=\text { prot } \\
\text { ulmonar }\end{array}$ & $\begin{array}{l}\text { mber of } \\
\text { D); } \mathrm{C}=\mathrm{ti} \\
\text { gain, ede } \\
\text { uria, alb } \\
\text { dema, d }\end{array}$ & $\begin{array}{l}\text { es (tota } \\
\text { of mate } \\
\text { E = hy } \\
\text { inuria; } \\
\text { noe; J }=\end{array}$ & $\begin{array}{l}\mathrm{n}= \\
\mathrm{c} \text { nal } \\
\text { per- } \\
\mathrm{H}= \\
\text { ele- }\end{array}$ & $\begin{array}{l}\text { vated liver } \\
\text { ea, vomit } \\
\text { ation } \pm \\
Q=\text { surviv } \\
1 \text { Thre }\end{array}$ & $\begin{array}{l}\text { enzyme } \\
\text { ng; } \mathrm{M}= \\
\mathrm{D}) ; \mathrm{O}= \\
\text { al; } \mathrm{R}=\mathrm{ti} \\
\text { cases pc }\end{array}$ & $\begin{array}{l}\mathrm{K}=\text { oliq } \\
\text { hrombo } \\
\text { ntrauter } \\
\text { ne until } \\
\text { stpartun }\end{array}$ & $\begin{array}{l}\text { uria; } L= \\
\text { ytopeni } \\
\text { ne deatl } \\
\text { naternal } \\
{ }^{2} \text { One }\end{array}$ & $\begin{array}{l}\text { headach } \\
\mathrm{N}=\text { tin } \\
\text { still birt } \\
\text { ceconval } \\
\text { se post }\end{array}$ & $\begin{array}{l}\text { visual } \\
\text { of deli } \\
\text { P }=\mathrm{d} \\
\text { scence } \\
\text { artum. }\end{array}$ & $\begin{array}{l}\text { isturbar } \\
\text { ery (wee } \\
\text { th after } \\
\text { ays } \pm s\end{array}$ & $\begin{array}{l}\text { es, nau- } \\
\text { s of ges- } \\
\text { delivery; } \\
\text { ). }\end{array}$ \\
\hline
\end{tabular}


albuminuria and proteinuria (42.9\%), elevated uric acid and creatinine (25\%), mild elevated liver enzymes (19.6\%), oliguria (16.1\%) and headache and visual disturbances (14.3\%). Severe maternal complications including pulmonary edema occurred in $21.4 \%$ of cases. The average rate of intrauterine death and stillbirth was $35.7 \%$ and the average time until maternal symptoms disappeared was 8.9 days.

\section{Discussion}

The present case of a fetal congenital heart disorder (Ebstein's anomaly) is a typical example of how fetal symptoms of severe hydrops and subcutaneous edema

\begin{tabular}{|c|c|c|c|c|}
\hline \multicolumn{4}{|c|}{ Fetal outcome } & \multirow[t]{2}{*}{$\mathrm{R}$} \\
\hline $\mathrm{N}$ & $\mathrm{O}$ & $\mathrm{P}$ & Q & \\
\hline $\begin{array}{l}29.6 \pm \\
2.7\end{array}$ & $\begin{array}{l}8 \\
(53.3)\end{array}$ & $\begin{array}{l}4 \\
(26.7)\end{array}$ & $\begin{array}{l}2 \\
(13.3)\end{array}$ & $\begin{array}{l}4.8 \pm \\
4.5\end{array}$ \\
\hline $\begin{array}{l}30.5 \pm \\
7.6 \\
26.8 \pm \\
6.3 \\
36.7 \pm \\
5.8\end{array}$ & $\begin{array}{l}3 \\
(30)\end{array}$ & $\begin{array}{l}2 \\
(20)\end{array}$ & $\begin{array}{l}5 \\
(50)\end{array}$ & $\begin{array}{l}10.7 \pm \\
7.3\end{array}$ \\
\hline $\begin{array}{l}30.6 \pm \\
5 \\
31.0 \pm \\
6.2 \\
30.5 \pm \\
0.7 \\
28.0\end{array}$ & $\begin{array}{l}5 \\
(55.6)\end{array}$ & $\begin{array}{l}1 \\
(11.1)\end{array}$ & $\begin{array}{l}3 \\
(33.3)\end{array}$ & $\begin{array}{l}13.5 \pm \\
4.9\end{array}$ \\
\hline $\begin{array}{l}30.8 \pm \\
4.4 \\
33.2 \pm \\
4.1 \\
30.6 \pm \\
2.7 \\
24.0 \pm \\
3.5 \\
35.5 \pm \\
0.7 \\
30.4 \pm \\
3.2\end{array}$ & $\begin{array}{l}4 \\
(19)\end{array}$ & $\begin{array}{l}7 \\
(33.3)\end{array}$ & $\begin{array}{l}10 \\
(47.6)\end{array}$ & $\begin{array}{l}6.7 \pm \\
5.3\end{array}$ \\
\hline
\end{tabular}

Mirror Syndrome can 'mirror' and can cause maternal complications. In this case the patient presented preeclampsia-like symptoms with severe edema, mild elevated liver enzymes and mild anemia without hypertension or decreased platelets. In addition, the patient developed a pulmonary infection on top of severe pleural effusion and presented right cardiac failure with pericardial effusion.

The etiology of the mirror syndrome remains unclear and a review of 56 cases reported in the literature until December 2008 presents a wide variety of fetal causes associated with mirror syndrome (table 1). In most of the cases (29\%) severe rhesus isoimmunization in association with the mirror syndrome has been reported. Besides these immunological complications, mirror syndrome was reported in cases with twin pregnancies $(\mathrm{n}=10)$ and viral infections (parvovirus B19 $\mathrm{n}=6$, cytomegalovirus $\mathrm{n}=2$, Coxsackie $\mathrm{B}$ virus $\mathrm{n}=1$ ). Others reported cases of placenta anomalies such as placental tumors $(n=3)$, cases of sacrococcygeal teratoma $(n=3)$, aneurysm of the vein of Galen $(n=1)$, stenosis of the aorta $(n=1)$ and Ebstein's anomaly $(\mathrm{n}=2)$. 'Toxic placental agents' were suspected to be involved in the mirror syndrome. Increased levels of angiogenic factors (sFLT1, sENG) in a case of CMV infection [19, 20], as well as elevated IL-6 level seemed to be involved [5]. For a review of the pathophysiological hypotheses, see Carbillon et al. [6].

The mirror syndrome which is related to fetal hydrops and large placental mass has several clinical characteristics, but maternal edema as in our case seems to be a key symptom (89.3\%). Further markers such as mild anemia and hemodilution (46.4\%), mild elevated liver enzymes (19.6\%) and pulmonary edema (21.4\%) were reported (table 3). Other indicators found in the literature such as elevated blood pressure (60.7\%), albuminuria and proteinuria (42.9\%), elevated uric acid and creatinine (25\%), oliguria (16.1\%), headache and visual disturbances (14.3\%) and low platelets (7.1\%) were not present in our case.

The problem of distinguishing between mirror syndrome and preeclampsia is obvious. Maternal symptoms included a wide variety. Several authors have tried to differentiate between the mirror syndrome and preeclampsia, however, since the incidence of the mirror syndrome is low, no large series of patients have been published. This is the first complete review of mirror syndrome, which includes 56 cases reported in the literature. In the present review, characteristics which were reported to be of value to differentiate between mirror syndrome and preeclampsia such as generalized pruritus $(\mathrm{n}=2)$, formation of abdominal blebs $(n=1)$, and the absence of hyper- 
active reflexes $(n=1)$ were found only in very few cases (table 1). Hemoconcentration is a typical pathophysiological feature in preeclampsia [21]. Reviewing the available cases in the literature (table 1), the consistent reporting of observation of low maternal hematocrit concentrations and mild anemia as the third most common symptom (46.6\% of all reported cases) seems to be capable of differentiating the mirror syndrome from preeclampsia as suggested before $[5,6]$. Further symptoms such as elevated serum uric acid or creatinine levels were only reported in $25 \%$ of all cases (table 3). Regardless of the fetal cause, maternal hypertension was the second most common symptom and illustrates the difficulty to distinguish mirror syndrome from preeclampsia.

Once the cause is treated and the fetal hydrops improves, maternal symptoms might disappear as described in several cases. Allen et al. [51] published a case of fetal pelvic mass which resulted in bladder outlet obstruction, subsequent bladder rupture and massive urinary ascites with thoracic and cardiac compression, and subsequent hydrops fetalis. Placement of a peritoneal-amniotic shunt resolved the fetal hydrops and maternal mirror syndrome [22]. In another case, hypertension and severe edema of the mother resolved after selective termination of the hydropic fetus in a twin-twin transfusion syndrome [23, 24]. Maternal treatment with diuretics, calcium channel blockers and $\beta$-blockers in a case of mirror syndrome due to parvovirus B19 complicated by severe maternal pulmonary effusion resolved maternal symptoms [13]. Ad- equate treatment of fetal tachycardia in fetuses with hydrops due to cardiac failure resulted in an improvement of fetal heart function, reduction in fetal hydrops and maternal edema $[25,26]$.

Reviewing the literature, maternal symptoms associated with mirror syndrome disappeared shortly after the successful treatment of fetal symptoms or the termination of pregnancy (mean 8.9 days). Treatment of hydrops fetalis depends on the cause. In our case, maternal therapy with methyldigoxin - described before to be possibly successful in the treatment of Ebstein's anomaly [18] unfortunately did not improve fetal cardiac function and did not reduce fetal hydrops or maternal symptoms. Compared to the two cases of Ebstein's anomaly associated with mirror syndrome found in the literature [6,27], labor induction was required due to the worsening of maternal symptoms and the newborn died few minutes after birth.

In conclusion, this case of Ebstein's anomaly and mirror syndrome demonstrates how fetal symptoms of cardiac failure, fetal hydrops and edema can be mirrored into maternal symptoms. Maternal edema and hemodilution associated with hydrops fetalis should alert physicians of this entity as it is associated with increased fetal mortality and maternal morbidity. However, the number of reported cases of mirror syndrome are limited. Further studies, both on the role of the placenta and the pathophysiology, are compulsory.

\section{References}

$>1$ Dunn PM: Dr John Ballantyne (1861-1923): perinatologist extraordinary of Edinburgh. Arch Dis Child 1993;68:66-67.

2 Ballantyne JW: The disease and deformities of the fetus. Edinburgh, Oliver \& Boyd, 1892.

$>3$ Reiss HE: Historical insights: John William Ballantyne 1861-1923. Hum Reprod Update 1999;5:386-389.

4 Kaiser IH: Ballantyne and triple edema. Am J Obstet Gynecol 1971;110:115-120.

5 Van Selm M, Kanhai HH, Gravenhorst JB: Maternal hydrops syndrome: a review. Obstet Gynecol Surv 1991;46:785-788.

6 Carbillon L, Oury JF, Guerin JM, Azancot A, Blot P: Clinical biological features of Ballantyne syndrome and the role of placental hydrops. Obstet Gynecol Surv 1997;52:310314.

7 Goeden AM, Worthington D: Spontaneous resolution of mirror syndrome. Obstet Gynecol 2005;106:1183-1186.
$>8$ Kumar B, Nazaretian SP, Ryan AJ, Simpson $>13$ Brochot C, Collinet P, Provost N, Subtil D: I: Mirror syndrome: a rare entity. Pathology Mirror syndrome due to parvovirus B19 hy2007;39:373-375.

$\checkmark 9$ Ordorica SA, Marks F, Frieden FJ, Hoskins IA, Young BK: Aneurysm of the vein of Galen: a new cause for Ballantyne syndrome. Am J Obstet Gynecol 1990;162:1166-1167.

-10 Finamore PS, Kontopoulos E, Price M, Giannina G, Smulian JC: Mirror syndrome associated with sacrococcygeal teratoma: a case report. J Reprod Med 2007;52:225-227.

11 Dorman SL, Cardwell MS: Ballantyne syndrome caused by a large placental chorioangioma. Am J Obstet Gynecol 1995;173:16321633.

$>12$ Midgley DY, Harding K: The mirror syndrome. Eur J Obstet Gynecol Reprod Biol 2000;88:201-202. drops complicated by severe maternal pulmonary effusion. Prenat Diagn 2006;26: 179-180.

14 Ville Y, de Gayffier A, Brivet F, Leruez M, Marchal P, Morinet F, Troalen F, Fernandez H, Frydman R: Fetal-maternal hydrops syndrome in human parvovirus infection. Fetal Diagn Ther 1995;10:204-206.

15 Ambrosini G, Nanhorngue K, Pascoli I, Cester M, Cosmi E: Mirror syndrome due to Coxsackie B virus associated to maternal peripartum cardiomyopathy. J Perinat Med 2008;36:453-454.

16 Chaoui R, Bollmann R, Hoffmann H, Zienert A, Bartho S: Ebstein anomaly as a rare prenatal diagnosis using Doppler echocardiography (in German). Klin Pädiatr 1990; 202:173-175. cause of a non-immunological fetal hydrops:
Braun/Brauer/Fuchs/Czernik/ Dudenhausen/Henrich/Sarioglu 
17 Hornberger LK, Sahn DJ, Kleinman CS, Copel JA, Reed KL: Tricuspid valve disease with significant tricuspid insufficiency in the fetus: diagnosis and outcome. J Am Coll Cardiol 1991;17:167-173.

- 18 Hsieh YY, Lee CC, Chang CC, Tsai HD, Yeh LS, Tsai CH: Successful prenatal digoxin therapy for Ebstein's anomaly with hydrops fetalis. A case report. J Reprod Med 1998;43: 710-712.

19 Rana S, Venkatesha S, DePaepe M, Chien EK, Paglia M, Karumanchi SA: Cytomegalovirus-induced mirror syndrome associated with elevated levels of circulating antiangiogenic factors. Obstet Gynecol 2007;109:549552.

20 Stepan H, Faber R: Cytomegalovirus-induced mirror syndrome associated with elevated levels of angiogenic factors. Obstet Gynecol 2007;109:1205-1206.

21 Redman CW: Hypertension in pregnancy: a case discussion. Kidney Int 1987;32:151-160.

-22 Livingston JC, Malik KM, Crombleholme TM, Lim FY, Sibai BM: Mirror syndrome: a novel approach to therapy with fetal peritoneal-amniotic shunt. Obstet Gynecol 2007; 110:540-543.

23 Heyborne KD, Chism DM: Reversal of Ballantyne syndrome by selective second-trimester fetal termination. A case report. J Reprod Med 2000;45:360-362.

-24 Heyborne KD, Porreco RP: Selective fetocide reverses preeclampsia in discordant twins. Am J Obstet Gynecol 2004;191:477-480.

-25 Frohn-Mulder IM, Stewart PA, Witsenburg M, Den Hollander NS, Wladimiroff JW, Hess J: The efficacy of flecainide versus digoxin in the management of fetal supraventricular tachycardia. Prenat Diagn 1995;15: 1297-1302.

26 Simpson JM, Sharland GK: Fetal tachycardias: management and outcome of 127 consecutive cases. Heart 1998;79:576-581.

27 Leung WC, Lam HS, Tang MH, Lao TT: Biochemical hypothyroidism - a new finding in mirror syndrome? Eur J Obstet Gynecol Reprod Biol 2006;125:269-271.

28 Cohen A: Maternal syndrome in Rh iso-immunization: report of a case. J Obstet Gynaecol Br Emp 1960;67:325-327.

29 De Silva K, Fonseka MN: Rhesus iso-immunization with a complex maternal syndrome. A case report of a patient with hydrops foetalis, pregnancy, toxaemia, large placenta and bilateral theca-lutein cysts with torsion of one ovary. Ceylon Med J 1971;16:178-181.

- 30 Hirsch MR, Mark MS: Pseudotoxemia and erythroblastosis. Report of a case. Obstet Gynecol 1964;24:47-48.

31 John AH, Duncan AS: The maternal syndrome associated with hydrops foetalis. J Obstet Gynaecol Br Commonw 1964;71:6165.
32 Keane B: Maternal syndrome associated with hydrops fetalis: case report. NZ Med J 1978; $88: 56-57$.

33 Lobato G, Nakamura-Pereira M: Reversion of the Ballantyne syndrome despite fetal hydrops persistence. Fetal Diagn Ther 2008;24: 474-477.

34 Mahmood TA: A reappraisal of the maternal syndrome associated with hydrops fetalis. Eur J Obstet Gynecol Reprod Biol 1987;25: 159-163.

35 Nicolay KS, Gainey HL: Pseudotoxemic state associated with severe $\mathrm{Rh}$ isoimmunization. Am J Obstet Gynecol 1964;89:41-45.

36 O’Driscoll DT: A fluid retention syndrome associated with severe iso-immunization to the rhesus factor. J Obstet Gynaecol Br Emp 1956;63:372-374

37 Rustamov O: PO02.85: mirror syndrome: a case report. Ultrasound Obstet Gynecol 2006;28:543

38 Pirhonen JP, Hartgill TW: Spontaneous reversal of mirror syndrome in a twin pregnancy after a single fetal death. Eur J Obstet Gynecol Reprod Biol 2004;116:106-107.

39 Hayashi S, Sago H, Hayashi R, Nakagawa S, Kitagawa M, Miyasaka K, Chiba T, Natori M: Manifestation of mirror syndrome after fetoscopic laser photocoagulation in severe twin-twin transfusion syndrome. Fetal Diagn Ther 2006;21:51-54

40 Matsubara S, Ohmaru T, Ohkuchi A, Arai F, Kuwata T, Usui R, Takahashi K, Izumi A, Watanabe T, Suzuki M: Mirror syndrome associated with hydropic acardius in triplet pregnancy. Fetal Diagn Ther 2008;24:429433.

41 Olivella A, Martinez JM, Lopez M, Del Rio M, Bennasar M, Peurto B, Coll O, Gratacos E: PO5.07: Two cases of mirror syndrome in TTTS with and without fetoscopic treatment. Ultrasound Obstet Gynecol 2006; 28:561.

42 Chang YL, Chao AS, Hsu JJ, Chang SD, Soong YK: Selective fetocide reversed mirror syndrome in a dichorionic triplet pregnancy with severe twin-twin transfusion syndrome: a case report. Fetal Diagn Ther 2007; 22:428-430.

43 Matsubara M, Nakata M, Murata S, Miwa I, Sumie M, Sugino N: Resolution of mirror syndrome after successful fetoscopic laser photocoagulation of communicating placental vessels in severe twin-twin transfusion syndrome. Prenat Diagn 2008;28:11671168 .

44 Quagliarello JR, Passalaqua AM, Greco MA, Zinberg S, Young BK: Ballantyne's triple edema syndrome: prenatal diagnosis with ultrasound and maternal renal biopsy findings. Am J Obstet Gynecol 1978;132:580-581.
45 Duthie SJ, Walkinshaw SA: Parvovirus associated fetal hydrops: reversal of pregnancyinduced proteinuric hypertension by in utero fetal transfusion. Br J Obstet Gynaecol 1995;102:1011-1013.

-46 Proust S, Philippe HJ, Paumier A, Joubert M, Boog G, Winer N: Mirror preeclampsia: Ballantyne's syndrome. Two cases (in French). J Gynecol Obstet Biol Reprod (Paris) 2006;35: 270-274.

47 Mizrahi-Arnaud A, Wilkins Haug L, Marshall A, Silva V: Maternal mirror syndrome after in utero aortic valve dilation. A case report. Fetal Diagn Ther 2006;21:439-443.

48 Wurm D, Loffler G, Lindinger A, Gortner L: Congenital disorders of glycosylation type Ia as a cause of mirror syndrome. J Perinatol 2007;27:802-804.

49 Paternoster DM, Manganelli F, Minucci D, Nanhorngue KN, Memmo A, Bertoldini M, Nicolini U: Ballantyne syndrome: a case report. Fetal Diagn Ther 2006;21:92-95.

50 Valsky DV, Daum H, Yagel S: Reversal of mirror syndrome after prenatal treatment of Diamond-Blackfan anemia. Prenat Diagn 2007;27:1161-1164.

51 Allen AT, Dress AF, Moore WF: Mirror syndrome resulting from metastatic congenital neuroblastoma. Int J Gynecol Pathol 2007; 26:310-312.

-52 Gherman RB, Incerpi MH, Wing DA, Goodwin TM: Ballantyne syndrome: is placental ischemia the etiology? J Matern Fetal Med 1998;7:227-229.

53 Galimberti A, Jain S: Placental chorioangioma as a cause of maternal hydrops syndrome. J Obstet Gynaecol 2000;20:91

54 Deurloo K, Devlieger R, Oepkes D: Maternal hydrops syndrome following successful treatment of fetal hydrops by shunting of bilateral hydrothorax. Prenat Diagn 2003;23: 944-945.

55 Ibele A, Flake A, Shaaban A: Survival of a profoundly hydropic fetus with a sacrococcygeal teratoma delivered at 27 weeks of gestation for maternal mirror syndrome. J Pediatr Surg 2008;43:e17-e20.

56 Nakamura K, Itoh H, Sagawa N, Kakui K, Nakayama T, Yamada S, Fujii S: A case of peripartum cardiomyopathy with a transient increase of plasma interleukin- 6 concentration occurred following mirror syndrome. J Perinat Med 2002;30:426-428.

57 Vidaeff AC, Pschirrer ER, Mastrobattista JM, Gilstrap LC 3rd, Ramin SM: Mirror syndrome. A case report. J Reprod Med 2002;47: 770-774.

58 Vidaeff AC, Ross PJ, Livingston CK, Parks DH: Gigantomastia complicating mirror syndrome in pregnancy. Obstet Gynecol 2003;101:1139-1142. 